\title{
Vacuum solutions of the gravitational field equations in the brane world model
}

\author{
T. Harko* and M. K. Mak ${ }^{\dagger}$ \\ Department of Physics, The University of Hong Kong, Pokfulam Road, Hong Kong
}

(Received 6 October 2003; published 18 March 2004)

\begin{abstract}
We consider some classes of solutions of the static, spherically symmetric gravitational field equations in the vacuum in the brane world scenario, in which our Universe is a three-brane embedded in a higher dimensional space-time. The vacuum field equations on the brane are reduced to a system of two ordinary differential equations, which describe all the geometric properties of the vacuum as functions of the dark pressure and dark radiation terms (the projections of the Weyl curvature of the bulk, generating nonlocal brane stresses). Several classes of exact solutions of the vacuum gravitational field equations on the brane are derived. In the particular case of a vanishing dark pressure, the integration of the field equations can be reduced to the integration of an Abel type equation. A perturbative procedure, based on the iterative solution of an integral equation, is also developed for this case. Brane vacuums with particular symmetries are investigated by using Lie group techniques. In the case of a static vacuum brane admitting a one-parameter group of conformal motions, the exact solution of the field equations can be found, with the functional form of the dark radiation and pressure terms uniquely fixed by the symmetry. The requirement of the invariance of the field equations with respect to the quasihomologous group of transformations also imposes a unique, linear proportionality relation between the dark energy and dark pressure. A homology theorem for the static, spherically symmetric gravitational field equations in the vacuum on the brane is also proven.
\end{abstract}

DOI: 10.1103/PhysRevD.69.064020

PACS number(s): 04.50.+h, 04.20.Cv, 04.20.Jb

\section{INTRODUCTION}

The idea, proposed in [1], that our four-dimensional Universe might be a three-brane, embedded in a fivedimensional space-time (the bulk), has attracted considerable interest in the past few years. According to the brane-world scenario, the physical fields (electromagnetic, Yang-Mills, etc.) in our four-dimensional Universe are confined to the three-brane. These fields are assumed to arise as fluctuations of branes in string theories. Only gravity can freely propagate in both the brane and bulk space-times, with the gravitational self-couplings not significantly modified. This model originated from the study of a single 3-brane embedded in five dimensions, with the 5D metric given by $d s^{2}$ $=e^{-f(y)} \eta_{\mu \nu} d x^{\mu} d x^{\nu}+d y^{2}$, which, due to the appearance of the warp factor, could produce a large hierarchy between the scale of particle physics and gravity. Even if the fifth dimension is uncompactified, standard 4D gravity is reproduced on the brane. Hence this model allows the presence of large, or even infinite noncompact extra dimensions. Our brane is identified to a domain wall in a 5-dimensional anti-de Sitter space-time.

The Randall-Sundrum model was inspired by superstring theory. The ten-dimensional $E_{8} \times E_{8}$ heterotic string theory, which contains the standard model of elementary particles, could be a promising candidate for the description of the real Universe. This theory is connected with an elevendimensional theory, $M$ theory, compactified on the orbifold $R^{10} \times S^{1} / Z_{2}$ [2]. In this model we have two separated tendimensional manifolds. For a review of dynamics and geometry of brane Universes see [3].

\footnotetext{
*Electronic address: harko@hkucc.hku.hk

†Electronic address: mkmak@vtc.edu.hk
}

Due to the correction terms coming from the extra dimensions, significant deviations from the Einstein theory occur in brane world models at very high energies [4,5]. Gravity is largely modified at the electroweak scale $1 \mathrm{TeV}$. The cosmological implications of the brane world theories have been extensively investigated in the physical literature [6]. Gravitational collapse can also produce high energies, with the five dimensional effects playing an important role in the formation of black holes [7].

For standard general relativistic spherical compact objects the exterior space-time is described by the Schwarzschild metric. In the five dimensional brane world models, the high energy corrections to the energy density, together with the Weyl stresses from bulk gravitons, imply that on the brane the exterior metric of a static star is no longer the Schwarzschild metric [8]. The presence of the Weyl stresses also means that the matching conditions do not have a unique solution on the brane; the knowledge of the five-dimensional Weyl tensor is needed as a minimum condition for uniqueness. Static, spherically symmetric exterior vacuum solutions of the brane world models have been proposed first by Dadhich et al. [8] and Germani and Maartens [9]. The first of these solutions, obtained in [8], has the mathematical form of the Reissner-Nördstrom solution, in which a tidal Weyl parameter plays the role of the electric charge of the general relativistic solution. The solution has been obtained by imposing the null energy condition on the 3-brane for a bulk having nonzero Weyl curvature. The solution can be matched to the interior solution corresponding to a constant density brane world star. A second exterior solution, which also matches a constant density interior, has been derived in [9].

Two families of analytic solutions of the spherically symmetric vacuum brane world model equations (with $g_{t t}$ $\left.\neq-1 / g_{r r}\right)$, parametrized by the Arnowitt-Deser-Misner $(\mathrm{ADM})$ mass and a parametrized post-Newtonian (PPN) pa- 
rameter $\beta$, have been obtained by Casadio, Fabri and Mazzacurati [10]. Nonsingular black-hole solutions in the brane world model have been considered in [11], by relaxing the condition of the zero scalar curvature but retaining the null energy condition. The "on brane" 4-dimensional Gauss and Codazzi equations for an arbitrary static spherically symmetric star in a Randall-Sundrum type II brane world have been completely solved by Visser and Wiltshire [12]. The onbrane boundary can be used to determine the full 5-dimensional space-time geometry. The procedure can be generalized to solid objects such as planets. A method to extend into the bulk asymptotically flat static spherically symmetric brane-world metrics has been proposed by Casadio and Mazzacurati [13]. The exact integration of the field equations along the fifth coordinate was done by using the multipole $(1 / r)$ expansion. The results show that the shape of the horizon of the brane black hole solutions is very likely a flat "pancake" for astrophysical sources.

Stellar structure in brane worlds is very different from that in ordinary general relativity. An exact interior uniformdensity stellar solution on the brane has been found in [9]. In this model the general relativistic upper bound for the massradius ratio, $M / R<4 / 9$, is reduced by 5 -dimensional highenergy effects. The existence of brane world neutron stars leads to a constraint on the brane tension, which is stronger than the big-bang nucleosynthesis constraint, but weaker than the Newton-law experimental constraints [9].

It is the purpose of the present paper to systematically consider spherically symmetric space-times in vacuum on the brane. As a first step we derive the two basic ordinary differential equations for the dark radiation and dark pressure, describing the geometry of the vacuum on the brane. By means of some appropriate transformations these equations take the form of an autonomous system of two ordinary differential equations. Some simple integrability cases are considered, leading to some already known or new vacuum solutions on the brane. The very important case corresponding to a vanishing dark pressure term is considered in detail. The integration of the gravitational field equations in the vacuum on the brane is reduced to the integration of an Abel type equation. Since this equation does not satisfy the known integrability conditions, the solution is obtained in terms of perturbative series obtained by solving the integral equation associated to this problem.

Next we consider vacuum space-times on the brane that are related to some particular Lie groups of transformations. As a first group of admissible transformations for the vacuum on the brane we shall consider spherically symmetric and static solutions of the gravitational field equations that admit a one-parameter group of conformal motions, i.e., the metric tensor $g_{\mu \nu}$ has the property $L_{\xi} g_{\mu \nu}=\phi(r) g_{\mu \nu}$, where the left-hand side is the Lie derivative of the metric tensor, describing the gravitational field on the vacuum brane, with respect to the vector field $\xi^{\mu}$, and $\phi$ is an arbitrary function of the radial coordinate $r$. With this assumption the gravitational field equations describing the static vacuum brane can be integrated in Schwarzschild coordinates, and an exact simple solution, corresponding to a brane admitting a one-parameter group of motions, can be obtained.
Suppose that from some static, spherically symmetric solution of the vacuum gravitational field equations on the brane we have obtained we want to construct other physical solutions of the field equations by means of scale transformations. The process of constructing a new physical model by applying scale changes to the given initial model is referred to as a "homology transformation" [14]. The homology properties of stars have been intensively investigated in astrophysics, and families of stars constructed in such a way from a given star are called homologous stars. For Newtonian homologous stars in equilibrium, the individual members are related to each other by transformations of the form $r$ $\rightarrow \bar{r}=a r, \rho \rightarrow \bar{\rho}=b \rho$ and $M \rightarrow \bar{M}=c M$, with $a, b, c$ constants. Chandrasekhar [14] refers to this change of scale as a "homologous transformation," and the homology theorem of Chandrasekhar [14] states that if $\theta(\xi)$ is a solution of the stellar structure equations then so is $C^{2 /(n-1)} \theta(C \xi)$ also, where $C$ is an arbitrary constant and $1<n \leqslant 5$. By analyzing the homology transformation properties of the gravitational field equations on the static vacuum brane, by using Lie group theory techniques, we shall show that the requirement of the invariance of the field equations with respect to an infinitesimal generator $\mathbf{X}$ fixes in a unique way the relation between the dark pressure and the dark radiation terms. We also prove the homology theorem for the gravitational field equations in vacuum on the brane.

The present paper is organized as follows. The basic equations describing the spherically symmetric gravitational field equations in the vacuum on the brane are derived in Sec. II. Some particular classes of solutions for vacuum branes are obtained in Sec. III. In Sec. IV we consider vacuum brane space-times admitting a one parameter group of conformal motions. Homology properties of the gravitational field equations are investigated in Sec. V. We conclude and discuss our results in Sec. VI.

\section{STATIC, SPHERICALLY SYMMETRIC VACUUM FIELD EQUATIONS ON THE BRANE}

On the 5-dimensional space-time (the bulk), with the negative vacuum energy $\Lambda_{5}$ and brane energy-momentum as the source of the gravitational field, the Einstein field equations are given by

$$
\begin{aligned}
& G_{I J}=k_{5}^{2} T_{I J}, \\
& T_{I J}=-\Lambda_{5} g_{I J}+\delta(Y)\left[-\lambda_{b} g_{I J}+T_{I J}^{\text {matter }}\right],
\end{aligned}
$$

with $\lambda_{b}$ the vacuum energy on the brane and $k_{5}^{2}=8 \pi G_{5}$. In this space-time a brane is a fixed point of the $Z_{2}$ symmetry. In the following, capital Latin indices run in the range $0, \ldots, 4$, while Greek indices take the values $0, \ldots, 3$.

Assuming a metric of the form $d s^{2}=\left(n_{I} n_{J}\right.$ $\left.+g_{I J}\right) d x^{I} d x^{J}$, with $n_{I} d x^{I}=d \chi$ the unit normal to the $\chi$ $=$ const hypersurfaces and $g_{I J}$ the induced metric on $\chi$ $=$ const hypersurfaces, the effective four-dimensional gravitational equations on the brane (the Gauss equation) take the form $[4,5]$ 


$$
G_{\mu \nu}=-\Lambda g_{\mu \nu}+k_{4}^{2} T_{\mu \nu}+k_{5}^{4} S_{\mu \nu}-E_{\mu \nu},
$$

where $S_{\mu \nu}$ is the local quadratic energy-momentum correction

$$
S_{\mu \nu}=\frac{1}{12} T T_{\mu \nu}-\frac{1}{4} T_{\mu}{ }^{\alpha} T_{\nu \alpha}+\frac{1}{24} g_{\mu \nu}\left(3 T^{\alpha \beta} T_{\alpha \beta}-T^{2}\right),
$$

and $E_{\mu \nu}$ is the nonlocal effect from the free bulk gravitational field, the transmitted projection of the bulk Weyl tensor $C_{I A J B}, E_{I J}=C_{I A J B} n^{A} n^{B}$, with the property $E_{I J}$ $\rightarrow E_{\mu \nu} \delta_{I}^{\mu} \delta_{J}^{\nu}$ as $\chi \rightarrow 0$. We have also denoted $k_{4}^{2}=8 \pi G$, with $G$ the usual four-dimensional gravitational constant.

The four-dimensional cosmological constant, $\Lambda$, and the four-dimensional coupling constant, $k_{4}$, are given by $\Lambda$ $=k_{5}^{2}\left(\Lambda_{5}+k_{5}^{2} \lambda_{b}^{2} / 6\right) / 2$ and $k_{4}^{2}=k_{5}^{4} \lambda_{b} / 6$, respectively. In the limit $\lambda_{b}^{-1} \rightarrow 0$ we recover standard general relativity.

The Einstein equation in the bulk and the Codazzi equation also imply the conservation of the energy-momentum tensor of the matter on the brane, $D_{\nu} T_{\mu}{ }^{\nu}=0$, where $D_{\nu}$ denotes the brane covariant derivative. Moreover, from the contracted Bianchi identities on the brane it follows that the projected Weyl tensor should obey the constraint $D_{\nu} E_{\mu}{ }^{\nu}$ $=k_{5}^{4} D_{\nu} S_{\mu}{ }^{\nu}$.

The symmetry properties of $E_{\mu \nu}$ imply that in general we can decompose it irreducibly with respect to a chosen 4-velocity field $u^{\mu}$ as [3]

$$
E_{\mu \nu}=-k^{4}\left[U\left(u_{\mu} u_{\nu}+\frac{1}{3} h_{\mu \nu}\right)+P_{\mu \nu}+2 Q_{(\mu} u_{\nu)}\right],
$$

where $k=k_{5} / k_{4}, h_{\mu \nu}=g_{\mu \nu}+u_{\mu} u_{\nu}$ projects orthogonal to $u^{\mu}$, the "dark radiation" term $U=-k^{4} E_{\mu \nu} u^{\mu} u^{\nu}$ is a scalar, $Q_{\mu}=k^{4} h_{\mu}^{\alpha} E_{\alpha \beta}$ a spatial vector and $P_{\mu \nu}=-k^{4}\left[h_{(\mu}^{\alpha} h_{\nu)} \beta\right.$ $\left.-\frac{1}{3} h_{\mu \nu} h^{\alpha \beta}\right] E_{\alpha \beta}$ a spatial, symmetric and trace-free tensor.

In the case of the vacuum state with $\rho=p=0, T_{\mu \nu} \equiv 0$ and consequently $S_{\mu \nu}=0$. Therefore, by neglecting the effect of the cosmological constant, the field equations describing a static brane take the form

$$
R_{\mu \nu}=-E_{\mu \nu},
$$

with $R_{\mu}^{\mu}=0=E_{\mu}^{\mu}$. In the vacuum case $E_{\mu \nu}$ satisfies the constraint $D_{\nu} E_{\mu}{ }^{\nu}=0$. In an inertial frame at any point on the brane we have $u^{\mu}=\delta_{0}^{\mu}$ and $h_{\mu \nu}=\operatorname{diag}(0,1,1,1)$. In a static vacuum $Q_{\mu}=0$ and the constraint for $E_{\mu \nu}$ takes the form

$$
\frac{1}{3} D_{\mu} U+\frac{4}{3} U A_{\mu}+D^{\nu} P_{\mu \nu}+A^{\nu} P_{\mu \nu}=0
$$

where $D_{\mu}$ is the projection (orthogonal to $u^{\mu}$ ) of the covariant derivative and $A_{\mu}=u^{\nu} D_{\nu} u_{\mu}$ is the 4-acceleration. In the static spherically symmetric case we may choose $A_{\mu}$ $=A(r) r_{\mu}$ and $P_{\mu \nu}=P(r)\left(r_{\mu} r_{\nu}-\frac{1}{3} h_{\mu \nu}\right)$, where $A(r)$ and $P(r)$ (the "dark pressure") are some scalar functions of the radial distance $r$, and $r_{\mu}$ is a unit radial vector.

We chose the static spherically symmetric metric on the brane in the form

$$
d s^{2}=-e^{\nu(r)} d t^{2}+e^{\lambda(r)} d r^{2}+r^{2}\left(d \theta^{2}+\sin ^{2} \theta d \phi^{2}\right) .
$$

Then the gravitational field equations and the effective energy-momentum tensor conservation equation in the vacuum take the form

$$
\begin{aligned}
& -e^{-\lambda}\left(\frac{1}{r^{2}}-\frac{\lambda^{\prime}}{r}\right)+\frac{1}{r^{2}}=\frac{48 \pi G}{k^{4} \lambda_{b}} U, \\
& e^{-\lambda}\left(\frac{\nu^{\prime}}{r}+\frac{1}{r^{2}}\right)-\frac{1}{r^{2}}=\frac{16 \pi G}{k^{4} \lambda_{b}}(U+2 P), \\
& e^{-\lambda}\left(\nu^{\prime \prime}+\frac{\nu^{\prime 2}}{2}+\frac{\nu^{\prime}-\lambda^{\prime}}{r}-\frac{\nu^{\prime} \lambda^{\prime}}{2}\right)=\frac{32 \pi G}{k^{4} \lambda_{b}}(U-P), \\
& \nu^{\prime}=-\frac{U^{\prime}+2 P^{\prime}}{2 U+P}-\frac{6 P}{r(2 U+P)},
\end{aligned}
$$

where ${ }^{\prime}=d / d r$. Equation (8) can immediately be integrated to give

$$
e^{-\lambda}=1-\frac{C_{1}}{r}-\frac{Q(r)}{r},
$$

where $C_{1}$ is an arbitrary constant of integration, and we denoted

$$
Q(r)=\frac{48 \pi G}{k^{4} \lambda} \int r^{2} U(r) d r .
$$

The function $Q$ is the gravitational mass corresponding to the dark radiation term (the dark mass). For $U=0$ the metric coefficient given by Eq. (12) must tend to the standard general relativistic Schwarzschild metric coefficient, which gives $C_{1}=2 G M$, where $M=$ const is the mass of the gravitating body. In the following we also denote $\alpha$ $=16 \pi G / k^{4} \lambda$. By substituting $\nu^{\prime}$ given by Eq. (11) into Eq. (9) and with the use of Eq. (12) we obtain the following system of differential equations satisfied by the dark radiation term $U$, the dark pressure $P$ and the dark mass $Q$, describing the vacuum gravitational field, exterior to a massive body, in the brane world model:

$$
\begin{aligned}
& \frac{d U}{d r}=-\frac{(2 U+P)\left[2 G M+Q+\alpha(U+2 P) r^{3}\right]}{r^{2}\left(1-\frac{2 G M}{r}-\frac{Q}{r}\right)} \\
&-2 \frac{d P}{d r}-\frac{6 P}{r}, \\
& \frac{d Q}{d r}=3 \alpha r^{2} U .
\end{aligned}
$$

The system of Eqs. (14) and (15) can be transformed to an autonomous system of differential equations by means of the transformations 


$$
q=\frac{2 G M+Q}{r}, \quad \mu=3 \alpha r^{2} U, \quad p=3 \alpha r^{2} P, \quad \theta=\ln r .
$$

With the use of the new variable given by Eqs. (16), Eqs. (14) and (15) become

$$
\begin{aligned}
& \frac{d q}{d \theta}=\mu-q, \\
& \frac{d \mu}{d \theta}=-\frac{(2 \mu+p)\left[q+\frac{1}{3}(\mu+2 p)\right]}{1-q}-2 \frac{d p}{d \theta}+2 \mu-2 p .
\end{aligned}
$$

Equations (14) and (15), or, equivalently, Eqs. (17) and (18), may be called the structure equations of the vacuum on the brane.

\section{CLASSES OF VACUUM SOLUTIONS ON THE BRANE}

The system of structure equations (14), (15) is not closed until a further condition is imposed on the functions $U$ and $P$. By choosing some particular forms of these functions, several classes of static vacuum solutions can be generated in the framework of the brane world model. As a first case we consider that the dark radiation $U$ and the dark pressure $P$ satisfy the constraint

$$
2 U+P=0 .
$$

Then Eq. (14) takes the form

$$
\frac{d P}{d r}=-4 \frac{P}{r},
$$

with the general solution given by

$$
P=\frac{P_{0}}{r^{4}}, \quad U=-\frac{P_{0}}{2 r^{4}}
$$

where $P_{0}$ is an arbitrary constant of integration. Equation (15) immediately gives the dark mass as

$$
Q=\frac{3 \alpha P_{0}}{2 r}+Q_{0},
$$

where $Q_{0}$ is a constant of integration. Therefore the metric on the brane is

$$
e^{\nu}=e^{-\lambda}=1-\frac{2 G M+Q_{0}}{r}-\frac{3 \alpha P_{0}}{2 r^{2}} .
$$

This form of the metric has been first obtained by Dadhich et al. [8].

A second class of solutions of the system of Eqs. (14), (15) can be obtained by assuming that

$$
U+2 P=0 .
$$

Then Eq. (14) is transformed into an algebraic equation, which gives

$$
Q=\frac{2}{3} r-2 G M
$$

Hence the metric coefficients of the vacuum brane line element become

$$
e^{\nu}=C_{0} r^{2}, \quad e^{-\lambda}=\frac{1}{3},
$$

where $C_{0}$ is a constant of integration and $e^{\nu}$ has been obtained by integrating Eq. (11). For this class of solutions the projections of the Weyl bulk tensor are given by

$$
U=-2 P=\frac{2}{9 \alpha r^{2}} .
$$

In the case of a vanishing dark radiation, $U=0$, which also implies a vanishing dark mass $Q=0$, the dark pressure $P$ satisfies a Bernoulli type equation, given by

$$
\frac{d P}{d r}+\frac{3 P}{r}+\frac{P\left(G M+\alpha r^{3} P\right)}{r^{2}\left(1-\frac{2 G M}{r}\right)}=0,
$$

with the general solution

$$
P=\frac{1}{r^{3}\left(C_{1} \sqrt{\left.1-\frac{2 G M}{r}-\frac{\alpha}{G M}\right)}\right.},
$$

where $C_{1}$ is an arbitrary constant of integration. Equation (11) gives $\nu^{\prime}=-2 P^{\prime} / P-6 / r$, or $\exp (\nu)=C_{2} / P^{2} r^{6}$. Hence for $U=0$ the metric tensor components are given by

$$
\begin{aligned}
e^{\nu} & =C_{2}\left(1-\frac{2 G M}{r}\right)\left[C_{1}-\frac{\alpha}{G M}\left(1-\frac{2 G M}{r}\right)^{-1 / 2}\right]^{2}, \\
e^{-\lambda} & =1-\frac{2 G M}{r} .
\end{aligned}
$$

Since $\alpha / G M$ is very small, for a zero dark radiation, $U$ $=0$, the deviations from the Schwarzschild geometry are very small. The standard general relativistic result is recovered for $\alpha \rightarrow 0$, which gives $C_{1}^{2} C_{2}=1$.

Next we consider the case of a vanishing dark pressure $P=0$. The dark radiation and the dark mass can be obtained by solving the following system of coupled differential equations, which immediately follows from Eqs. (17) and (18):

$$
\begin{aligned}
& \frac{d q}{d \theta}=\mu-q \\
& \frac{d \mu}{d \theta}=2 \mu \frac{3-6 q-\mu}{3(1-q)} .
\end{aligned}
$$


By eliminating $\mu$ between Eqs. (31) and (32) we obtain the following second order differential equation for the function $q$ :

$$
3(1-q) \frac{d^{2} q}{d \theta^{2}}+(13 q-3) \frac{d q}{d \theta}+2\left(\frac{d q}{d \theta}\right)^{2}+2 q(7 q-3)=0
$$

By means of the successive transformations $d q / d \theta$ $=1 / v, v=w(1-q)^{-2 / 3}$, Eq. (33) can be transformed to the following, Abel type, first order differential equation:

$$
\begin{aligned}
\frac{d w}{d q} & -\frac{13 q-3}{3}(1-q)^{-5 / 3} w^{2} \\
& +\frac{2 q(3-7 q)}{3}(1-q)^{-7 / 3} w^{3}=0 .
\end{aligned}
$$

It is a matter of simple calculations to check that Eq. (34) has a particular solution of the form

$$
w=-\frac{1}{q}(1-q)^{2 / 3} \text {. }
$$

By introducing a new variable $\eta=(1-q)^{-1 / 3}, \quad q=1$ $-\eta^{-3}$, Eq. (34) can be further transformed to

$$
\frac{d w}{d \eta}-\frac{10 \eta^{3}-13}{\eta^{2}} w^{2}+\frac{2\left(\eta^{3}-1\right)\left(7-4 \eta^{3}\right)}{\eta^{3}} w^{3}=0 .
$$

Therefore we have reduced the problem of the integration of the gravitational field equations for the vacuum on the brane in the case of a vanishing dark pressure term, $P=0$, to the problem of the integration of an Abel type equation. However, Eq. (36) does not satisfy the standard integration conditions for Abel type equations [15], and an exact analytical solution of this equation seems to be difficult to obtain. Hence, in order to find some explicit solutions for the vacuum gravitational field on the brane, we have to use some perturbative methods.

By using the Laplace transform and the convolution theorem, the differential equation (33) is equivalent to the following integral equation:

$$
\begin{aligned}
q(\theta)= & \int_{\theta_{0}}^{\theta} F(\theta-x)\left[3 q \frac{d^{2} q}{d x^{2}}-13 q \frac{d q}{d x}-2\left(\frac{d q}{d x}\right)^{2}-14 q^{2}\right] d x \\
& +q_{0}(\theta)
\end{aligned}
$$

where

$$
\begin{aligned}
F(\theta-x) & =\frac{1}{9}\left[e^{2(\theta-x)}-e^{-(\theta-x)}\right], \\
q_{0}(\theta) & =A_{1} e^{-\theta}+A_{2} e^{2 \theta}
\end{aligned}
$$

and we denoted $A_{1}=\left[3 q\left(\theta_{0}\right)-\mu\left(\theta_{0}\right)\right] \exp \left(\theta_{0}\right) / 3$ and $A_{2}$ $=\mu\left(\theta_{0}\right) \exp \left(-2 \theta_{0}\right) / 3 . \theta_{0}=\ln r_{0}$ is an arbitrary point, like, for example, the vacuum boundary of a compact astrophysical object, in which the functions $q(r)$ and $\mu(r)$ take the values $q\left(r_{0}\right)=\left[2 G M+Q\left(r_{0}\right)\right] / r_{0}$ and $\mu\left(r_{0}\right)=3 \alpha r_{0}^{2} U\left(r_{0}\right)$, respectively.

The solution of the integral equation (37) can be easily obtained by using the method of successive approximations, or the method of iterations. In this way we can generate the solution to any desired degree of accuracy. Taking as an initial approximation the general solution of the linear part of the differential equation (33), the general solution of the integral equation (37) can be expressed in the first, second and $m$ th order approximation, $m \in N$, as follows:

$$
\begin{aligned}
q_{1}(\theta)= & \int_{\theta_{0}}^{\theta} F(\theta-x)\left[3 q_{0} \frac{d^{2} q_{0}}{d x^{2}}-13 q_{0} \frac{d q_{0}}{d x}\right. \\
& \left.-2\left(\frac{d q_{0}}{d x}\right)^{2}-14 q_{0}^{2}\right] d x+q_{0}(\theta) \\
q_{m}(\theta)= & \int_{\theta_{0}}^{\theta} F(\theta-x)\left[3 q_{m-1} \frac{d^{2} q_{m-1}}{d x^{2}}-13 q_{m-1} \frac{d q_{m-1}}{d x}\right. \\
& \left.-2\left(\frac{d q_{m-1}}{d x}\right)^{2}-14 q_{m-1}^{2}\right] d x+q_{m-1}(\theta) \\
q(\theta)= & \lim _{m \rightarrow \infty} q_{m}(\theta)
\end{aligned}
$$

The zeroth order approximation to the solution of the static spherically symmetric gravitational field equations in the vacuum on the brane is given by

$$
\begin{gathered}
e^{\nu}=\frac{C_{0}}{\sqrt{U}}=C_{0} \sqrt{\frac{\alpha}{A_{2}}}, \\
e^{-\lambda}=1-\frac{A_{1}}{r}-A_{2} r^{2}, \\
U=\frac{A_{2}}{\alpha},
\end{gathered}
$$

where $C_{0}$ is an arbitrary constant of integration.

The first order approximation to the solution is given by

$$
\begin{aligned}
e^{\nu}= & C_{0} \sqrt{\frac{\alpha r_{0}}{2}} \sqrt{\frac{r}{A_{2}\left(r_{0}-r\right)\left[A_{1}+A_{2} r_{0}^{2} r+A_{2} r_{0} r^{2}\right]}}, \\
e^{-\lambda}= & 1+\frac{A_{2} r_{0}^{2}\left[(4 / 5) A_{2} r_{0}^{3}+A_{1}\right]}{r}-3 A_{1} A_{2} r \\
& -2 A_{2}\left(2 A_{2} r_{0}^{2}-\frac{A_{1}}{r_{0}}\right) r^{2}+\frac{6}{5} A_{2}^{2} r^{4},
\end{aligned}
$$




$$
U=\frac{2 A_{2}\left(r_{0}-r\right)\left[A_{1}+A_{2} r_{0} r\left(r_{0}+r\right)\right]}{\alpha r_{0} r} .
$$

Therefore the general solution to the static gravitational field equations on the vacuum brane can be obtained in any order of approximation.

\section{STATIC VACUUM BRANES ADMITTING A ONE-PARAMETER GROUP OF CONFORMAL MOTIONS}

In the present section we are going to consider a special class of static vacuum brane solutions, which have as a group of admissible transformations the conformal motions.

For a spherically symmetric and static vacuum on the brane the assumption of the existence of a one-parameter group of conformal motions requires that the condition

$$
L_{\xi} g_{\mu \nu}=\xi_{\mu ; \nu}+\xi_{\nu ; \mu}=\phi(r) g_{\mu \nu}
$$

hold for the metric tensor components, where the left-hand side is the Lie derivative of the metric tensor, describing the vacuum brane gravitational field, with respect to the vector field $\xi^{\mu}$, and $\phi(r)$ is an arbitrary function of the radial coordinate $r$. We shall further restrict the field $\xi^{\mu}$ by demanding $\xi^{\mu} u_{\mu}=0$. Then as a consequence of the spherical symmetry we have $\xi^{2}=\xi^{3}=0$. This type of symmetry has been intensively used to describe the interior of neutral or charged general relativistic stellar-type objects [16]. With the assumption (49) the gravitational field equations describing the spherically symmetric static vacuum brane can be integrated in Schwarzschild coordinates and an exact solution can be obtained. Moreover, the requirement of the conformal invariance of the static brane uniquely fixes the functional form of the projections of the bulk Weyl tensor components $U(r)$ and $P(r)$.

Using the line element (7), Eq. (49) explicitly reads

$$
\begin{gathered}
\xi^{1} \nu^{\prime}=\phi, \quad \xi^{0}=\bar{C}=\text { const }, \\
\xi^{1}=\frac{\phi r}{2}, \quad \lambda^{\prime} \xi^{1}+2 \frac{d \xi^{1}}{d r}=\phi .
\end{gathered}
$$

Equations (50) have the general solution given by [16]

$$
e^{\nu}=A^{2} r^{2}, \quad \phi=C e^{-\lambda / 2}, \quad \xi^{\mu}=\bar{C} \delta_{0}^{\mu}+\delta_{1}^{\mu} \frac{\phi r}{2},
$$

with $A$ and $C$ arbitrary constants of integration.

Hence the requirement of the existence of conformal motions imposes strong constraints on the form of the metric tensor coefficients of the static vacuum brane. Substituting Eqs. (51) into the field equations (8)-(10) we obtain

$$
\begin{aligned}
& \frac{1}{r^{2}}\left(1-\frac{\phi^{2}}{C^{2}}\right)-\frac{2}{C^{2}} \frac{\phi \phi^{\prime}}{r}=3 \alpha U, \\
& \frac{1}{r^{2}}\left(1-3 \frac{\phi^{2}}{C^{2}}\right)=-\alpha(U+2 P),
\end{aligned}
$$

$$
\frac{1}{C^{2}} \frac{\phi^{2}}{r^{2}}+\frac{2}{C^{2}} \frac{\phi \phi^{\prime}}{r}=\alpha(U-P) .
$$

We can formally solve the field equations (53) and (54) to express $U$ and $P$ as

$$
\begin{aligned}
& U=\frac{1}{3 \alpha}\left[\frac{4}{C^{2}} \frac{\phi \phi^{\prime}}{r}-\frac{1}{r^{2}}\left(1-\frac{5}{C^{2}} \phi^{2}\right)\right], \\
& P=-\frac{1}{3 \alpha}\left[\frac{2}{C^{2}} \frac{\phi \phi^{\prime}}{r}+\frac{1}{r^{2}}\left(1-\frac{2}{C^{2}} \phi^{2}\right)\right] .
\end{aligned}
$$

With the use of Eqs. (52) and (55) it follows that the function $\phi$ satisfies the first order differential equation

$$
\frac{3}{C^{2}} \phi \phi^{\prime}=\frac{1}{r}\left(1-\frac{3}{C^{2}} \phi^{2}\right),
$$

with the general solution given by

$$
\phi^{2}=\frac{C^{2}}{3}\left(1+\frac{B}{r^{2}}\right)
$$

where $B>0$ is a constant of integration. Therefore the general solution of the static gravitational field equations on the brane for space-times admitting a one-parameter group of conformal motions is given by

$$
\begin{gathered}
e^{\nu}=A^{2} r^{2}, \quad e^{-\lambda}=\frac{1}{3}\left(1+\frac{B}{r^{2}}\right), \\
U=\frac{1}{9 \alpha r^{2}}\left(2+\frac{B}{r^{2}}\right), \\
P=\frac{1}{9 \alpha r^{2}}\left(\frac{4 B}{r^{2}}-1\right) .
\end{gathered}
$$

In the case $B=0$ we recover the solution given by Eqs. (26), satisfying the condition $U+2 P=0$. For this case the function $\phi=C / \sqrt{3}$ is a constant.

\section{HOMOLOGY PROPERTIES OF THE STATIC GRAVITATIONAL FIELD EQUATIONS IN VACUUM ON THE BRANE}

Let us assume that a solution of the field equations (8)(11) is known. Then it seems reasonable to require that a family of solutions should exist, whose individual members are related by more general transformations of the form $r$ $\rightarrow \bar{r}(r), U \rightarrow \bar{U}(U), P \rightarrow \bar{P}(P)$ and $Q \rightarrow \bar{Q}(Q)[18]$. We shall call a set of solutions of the vacuum gravitational field equations on the brane related by transformations of this form a homologous family of solutions. 
In order to obtain the homology properties of the structure equations (14) and (15), it is necessary first to close the system of equations. We shall do this by assuming that the dark pressure $P$ and the dark radiation $U$ terms are related by an arbitrary functional relation $P=P(U)$. Then, by denoting $\gamma(U)=P(U) / U$ and $d P / d U=P^{\prime}(U)=c_{s}$, the basic equations describing the vacuum gravitational field on the brane take the form

$$
\frac{d U}{d r}=-\frac{\gamma(U) U}{1+2 c_{s}} \frac{\left[1+2 \gamma^{-1}(U)\right]\left\{2 G M+Q+\alpha r^{3}[1+2 \gamma(U)] U\right\}+6 r-6(2 G M+Q)}{r^{2}\left(1-\frac{2 G M}{r}-\frac{Q}{r}\right)},
$$

$$
\frac{d Q}{d r}=3 \alpha r^{2} U
$$

A system of ordinary differential equations

$$
\frac{d y^{k}}{d x}=f^{k}(x, y), \quad k=1,2, \ldots, m,
$$

with $y=\left(y^{1}, y^{2}, \ldots, y^{m}\right)$ is invariant under the action of the infinitesimal generator $\mathbf{X}=\zeta(x, y)(\partial / \partial x)+\eta^{k}(x, y)\left(\partial / \partial y^{k}\right)$ if and only if $[\mathbf{L}, \mathbf{X}]=r \mathbf{X}$, where [] denotes the Lie bracket, $\mathbf{L}=(\partial / \partial x)+f^{k}\left(\partial / \partial y^{k}\right)$ and $r=\mathbf{L}(\zeta)[17]$, or, in explicit form [18],

$$
\begin{gathered}
\frac{\partial \eta^{k}}{\partial x}+f^{j} \frac{\partial \eta^{k}}{\partial y^{j}}-f^{k} \frac{\partial \zeta}{\partial x}-f^{k} f^{j} \frac{\partial \zeta}{\partial y^{j}}=\zeta \frac{\partial f^{k}}{\partial x}+\eta^{j} \frac{\partial f^{k}}{\partial y^{j}}, \\
k=1,2, \ldots, m .
\end{gathered}
$$

In the particular case where $\mathbf{X}$ generates quasihomologous transformations of the form $x \rightarrow \bar{x}(x), y^{j} \rightarrow \bar{y}^{j}\left(y^{j}\right)$ we have $\zeta=\zeta(x)$ and $\eta^{j}=\eta^{j}\left(y^{j}\right)$. As a result Eq. (65) becomes

$$
\frac{d \eta^{k}\left(y^{k}\right)}{d y^{k}}-\frac{d \zeta(x)}{d x}=\mathbf{X}\left(\ln \left|f^{k}\right|\right)
$$

with no sum over $k$.

To analyze the homologous behavior of Eqs. (62) and (63) with respect to quasihomologous transformations involving a general functional dependence of the physical parameters, $r$ $=\bar{r}(r), U=\bar{U}(U), P=\bar{P}(P)$ and $Q=\bar{Q}(Q)$, we shall investigate the group of transformations generated by the infinitesimal generator

$$
\mathbf{X}=\zeta(r) \frac{\partial}{\partial r}+\eta^{1}(U) \frac{\partial}{\partial U}+\eta^{2}(Q) \frac{\partial}{\partial Q} .
$$

As applied to the case of Eqs. (62) and (63), Eqs. (66) give

$$
\begin{aligned}
\frac{d \eta^{1}(U)}{d U}-\frac{d \zeta}{d r}= & \zeta(r) \frac{\partial}{\partial r} \ln \left\{\frac{\left[1+2 \gamma^{-1}\right]\left[2 G M+Q+\alpha r^{3}(1+2 \gamma) U\right]+6 r-6(2 G M+Q)}{r^{2}\left(1-\frac{2 G M}{r}-\frac{Q}{r}\right)}\right\} \\
& +\eta^{1}(U) \frac{\partial}{\partial U} \ln \left\{\gamma U \frac{\left[1+2 \gamma^{-1}\right]\left[2 G M+Q+\alpha r^{3}(1+2 \gamma) U\right]+6 r-6(2 G M+Q)}{1+2 c_{s}}\right\} \\
& +\eta^{2}(Q) \frac{\partial}{\partial Q} \ln \left\{\frac{\left[1+2 \gamma^{-1}\right]\left[2 G M+Q+\alpha r^{3}(1+2 \gamma) U\right]+6 r-6(2 G M+Q)}{\left(1-\frac{2 G M}{r}-\frac{Q}{r}\right)}\right\} \\
\frac{d \eta^{2}(Q)}{d Q}-\frac{d \zeta(r)}{d r}= & 2 \frac{\zeta(r)}{r}+\frac{\eta^{1}(U)}{U} .
\end{aligned}
$$

In Eq. (69) the variables can be easily separated, leading to general expressions for the functions $\zeta(r), \eta^{1}(U)$ and $\eta^{2}(Q)$ of the form

$$
\zeta(r)=\frac{a}{r^{2}}+b r, \quad \eta^{1}(U)=(c-3 b) U, \quad \eta^{2}(Q)=c Q+d,
$$

where $a, b, c, d$ are separation and integration constants, respectively. Substituting in Eq. (68) gives the following consistency condition for the coefficients $a, b, c, d$ and for the functions $\gamma(U)$ and $c_{s}$ : 


$$
\begin{aligned}
\frac{4 a}{r^{3}}+b= & (c-3 b) U\left(\frac{\gamma^{\prime}}{\gamma}-\frac{c_{s}^{\prime}}{1+c_{s}}\right)-\frac{a}{r^{4}} \frac{2 G M+Q}{1-\frac{2 G M}{r}-\frac{Q}{r}}+\frac{1}{r} \frac{(d-2 b G M)+(c-b) Q}{1-\frac{2 G M}{r}-\frac{Q}{r}} \\
& +\frac{a}{r^{2}} \frac{3 \alpha\left(1+2 \gamma^{-1}\right)(1+2 \gamma) U r^{2}+6}{\left(1+2 \gamma^{-1}\right)\left[2 G M+Q+\alpha(1+2 \gamma) U r^{3}\right]+6 r-6(2 G M+Q)} \\
& +\frac{\left(1+2 \gamma^{-1}\right)\left[3 \alpha b(1+2 \gamma) U r^{3}+c Q+d\right]+6 b r-6(c Q+d)}{\left(1+2 \gamma^{-1}\right)\left[2 G M+Q+\alpha(1+2 \gamma) U r^{3}\right]+6 r-6(2 G M+Q)} \\
& +(c-3 b) U \frac{-2 \gamma^{\prime} \gamma^{-2}\left[2 G M+Q+\alpha(1+2 \gamma) U r^{3}\right]+\alpha\left(1+2 \gamma^{-1}\right)\left(1+2 \gamma+2 \gamma^{\prime} U\right) r^{3}}{\left(1+2 \gamma^{-1}\right)\left[2 G M+Q+\alpha(1+2 \gamma) U r^{3}\right]+6 r-6(2 G M+Q)} .
\end{aligned}
$$

Equation (71) is identically satisfied for $a=b=c=d$ $=0$, corresponding to $\mathbf{X}=0$ (the identity transformation). In the second case, in order to satisfy the identity (71), we have to take as a first step $a=0$. Then we chose $d=2 b G M$ and $c=b$. From the general structure of the identity (71) it follows that it cannot be identically satisfied unless $\gamma^{\prime}=0$ and $c_{s}^{\prime}=0$, implying $\gamma=P / U=$ const and $\gamma=c_{s}=$ const. Then in order for Eq. (71) to be identically satisfied we must adopt for $b$ the value $b=1$ as the last compatibility condition. Therefore we have obtained the following theorem.

Theorem. The static, spherically symmetric gravitational field equations in vacuum on the brane are invariant with respect to the group of the quasihomologous transformations if and only if the dark pressure is proportional to the dark radiation, $P=\gamma U, \gamma=$ const.

The infinitesimal operator generating the group of quasihomologous transformations on the static brane has the form

$$
\mathbf{X}=r \frac{\partial}{\partial r}-2 U \frac{\partial}{\partial U}+(Q+2 G M) \frac{\partial}{\partial Q}
$$

The quantities $(Q+2 G M) / r$ and $U r^{2}$ (or any two independent functions of them) are homologous invariants. Hence the homology properties of the spherically symmetric vacuum space-times on the brane are described by the following homology theorem.

Homology Theorem. If $U(r)$ is a solution of the static, spherically symmetric gravitational field equations in vacuum on a brane with the dark pressure proportional to the dark radiation, then so also is $C^{2} U(C r)$, where $C$ is an arbitrary constant.

\section{DISCUSSIONS AND FINAL REMARKS}

In the present paper we have considered some properties of the vacuum exterior to compact astrophysical objects in the brane world model. The system of field equations can be reduced to two ordinary differential equations, in three unknowns, whose solution gives all the geometrical properties of the space-time. The system of basic equations describing the vacuum gravitational field equation on the brane is not uniquely determined and its solution depends on the func- tional relation between two unknown functions, the dark pressure $P$ and the dark radiation $U$. The symmetry properties of the vacuum brane space-times can uniquely fix the functional relation between these two free parameters of the model. The requirement that the vacuum on the brane admits a one-parameter group of conformal motions or a group of homologous transformations uniquely fixes the functional dependence of the free parameters $P$ and $U$. The relation between the dark pressure and the dark radiation for the vacuum on the brane admitting a one-parameter group of conformal motions is of the form $2 P+U=(B / \alpha) r^{-4}$. On the other hand the invariance of the field equations with respect to the Lie group of homologous transformations requires a linear proportionality relation between $P$ and $U, P$ $=\gamma U$. Once the relation between $P$ and $U$ is known, the general solution of the vacuum field equations can be found perturbatively.

The Schwarzschild solution is no longer the unique vacuum solution of the gravitational field equations. Moreover, most of the general solutions we have found are not asymptotically flat and consequently they are of a cosmological nature.

In order to obtain a manifestly coordinate invariant characterization of certain geometrical properties of geometries, such as for example curvature singularities, the Petrov type of the Weyl tensor, etc., the scalar invariants of the Riemann tensor have been extensively used. Two scalars, which have been considered in the physical literature, are the Kretschmann scalars, RiemSq $\equiv R_{i j k l} R^{i j k l}$ and RicciSq $\equiv R_{i j} R^{i j}$, where $R_{i j k l}$ is the Riemann curvature tensor.

For space-times which are the product of two 2-dimensional spaces, one Lorentzian and one Riemannian, subject to a separability condition on the function which couples the 2-spaces, it has been suggested [19] that the set

$$
C=\left\{R, r_{1}, r_{2}, w_{2}\right\},
$$

forms an independent set of scalar polynomial invariants, satisfying the number of degrees of freedom in the curvature. In Eq. (73) $R=g^{i l} g^{j k} R_{i j k l}$ is the Ricci scalar and the quantities $r_{1}, r_{2}$ and $w_{2}$ are defined according to [20] 


$$
\begin{aligned}
& r_{1}=\phi_{A B \dot{A} \dot{B}} \phi^{A B \dot{A} \dot{B}}=\frac{1}{4} S_{a}^{b} S_{b}^{a}, \\
& r_{2}=\phi_{A B \dot{A} \dot{B}} \phi_{C \dot{C}}^{B \dot{B}} \phi^{C A \dot{C} \dot{A}}=-\frac{1}{8} S_{a}^{b} S_{b}^{c} S_{c}^{a}, \\
& w_{2}=\Psi_{A B C D} \Psi_{E F}^{C D} \Psi^{E F A B}=-\frac{1}{8} \bar{C}_{a b c d} \bar{C}_{e f}^{c d} \bar{C}^{e f a b},
\end{aligned}
$$

where $S_{a}^{b}=R_{a}^{b}-\frac{1}{4} R \delta_{a}^{b}$ is the trace-free Ricci tensor, $\phi_{A B \dot{A} \dot{B}}$ denotes the spinor equivalent of $S_{a b}, \Psi_{A B C D}$ denotes the spinor equivalent of the Weyl tensor $C_{a b c d}$, and $\bar{C}_{a b c d}$ denotes the complex conjugate of the self-dual Weyl tensor, $C_{a b c d}^{+}=\frac{1}{2}\left(C_{a b c d}-i * C_{a b c d}\right)$.

In terms of the "electric" $E_{a c}=C_{a b c d} u^{b} u^{d}$ and "magnetic" $H_{a c}=C_{a b c d}^{*} u^{b} u^{d}$ parts of the Weyl tensor, where $u^{a}$ is a timelike unit vector and $C_{a b c d}^{*}=\frac{1}{2} \eta_{a b e f} C_{c d}^{e f}$ is the dual tensor, the invariant $w_{2}$ is given by [19]

$$
\begin{aligned}
w_{2}= & \frac{1}{32}\left(3 E_{b}^{a} H_{c}^{b} H_{a}^{c}-E_{b}^{a} E_{c}^{b} E_{a}^{c}\right) \\
& +\frac{i}{32}\left(H_{b}^{a} H_{c}^{b} H_{a}^{c}-3 E_{b}^{a} E_{c}^{b} H_{a}^{c}\right) .
\end{aligned}
$$

The values of the invariant set $\left\{R, r_{1}, r_{2}, w_{2}\right\}$ for some static spherically symmetric vacuum brane solutions are presented in the Appendix.

The corrections to the Newtonian potential on the brane have been considered by using perturbative expansions in the static weak-field regime. The leading order correction to the Newtonian potential on the brane is given by $\Phi=(G M / r)$ $\times\left(1+2 l^{2} / 3 r^{2}\right)[1]$, where $l$ is the curvature scale of the fivedimensional anti-de Sitter spacetime $\left(\mathrm{AdS}_{5}\right)$, or it can also involve a logarithmic factor [21]. This type of weak-field behavior cannot be recovered in the classes of solutions we have considered in the present paper. However, this could be possible for models involving a more precise knowledge of the general behavior of the dark radiation and dark pressure terms.

\section{APPENDIX}

In this appendix we present the values of the Kretschmann scalars $R i e m S q \equiv R_{i j k l} R^{i j k l}$ and $R i c c i S q \equiv R_{i j} R^{i j}$ and some values of the independent set of the scalar polynomial invariants $\left\{R, r_{1}, r_{2}, w_{2}\right\}$ for the exact static spherically symmetric vacuum brane geometries discussed in the paper.

(a)

$$
\begin{aligned}
e^{\nu} & =e^{-\lambda}=1-\frac{2 G M+Q_{0}}{r}-\frac{3 \alpha P_{0}}{2 r^{2}} \\
\text { RicciS } q & =\frac{9 \alpha^{2} P_{0}}{r^{8}}, \quad \text { Riem } q=\frac{6\left[21 \alpha^{2} P_{0}^{2}+12 \alpha P_{0}\left(2 G M+Q_{0}\right) r+2\left(2 G M+Q_{0}\right)^{2} r^{2}\right]}{r^{8}} \\
R & =0, \quad r_{1}=\frac{9 \alpha^{2} P_{0}}{4 r^{8}}, \quad r_{2}=0 \\
\operatorname{Re}\left(w_{2}\right) & =\frac{3\left[27 \alpha^{3} P_{0}^{3}+27 \alpha^{2} P_{0}^{2}\left(2 G M+Q_{0}\right) r+9 \alpha P_{0}\left(2 G M+Q_{0}\right)^{2} r^{2}+\left(2 G M+Q_{0}\right)^{3} r^{3}\right]}{4 r^{12}}, \quad \operatorname{Im}\left(w_{2}\right)=0 .
\end{aligned}
$$

(b)

$$
\begin{aligned}
e^{\nu} & =C_{2}\left(1-\frac{2 G M}{r}\right)\left[C_{1}-\frac{\alpha}{G M}\left(1-\frac{2 G M}{r}\right)^{-1 / 2}\right]^{2}, \quad e^{-\lambda}=1-\frac{2 G M}{r}, \\
\text { RicciS } q & =\frac{6 \alpha^{2}}{\left(\frac{\alpha}{G M}-C_{1} \sqrt{\left.1-\frac{2 G M}{r}\right)^{2}} r^{6}\right.} \\
\text { Riem } S q & =\frac{24\left[-4 C_{1}^{2} G^{3} M^{3}+\left(\alpha^{2}+2 C_{1}^{2} G^{2} M^{2}-2 \alpha C_{1} G M \sqrt{1-\frac{2 G M}{r}}\right)\right.}{\left(\frac{\alpha}{G M}-C_{1} \sqrt{1-\frac{2 G M}{r}}\right)^{2}} r^{7}
\end{aligned}
$$




$$
\begin{aligned}
& R=0, \quad r_{1}=\frac{3 \alpha^{2}}{2\left(\frac{\alpha}{G M}-C_{1} \sqrt{\left.1-\frac{2 G M}{r}\right)^{2} r^{6}}\right.}, \quad r_{2}=-\frac{3 \alpha^{3}}{4\left(\frac{\alpha}{G M}-C_{1} \sqrt{\left.1-\frac{2 G M}{r}\right)^{3} r^{9}}\right.}, \\
& \operatorname{Re}\left(w_{2}\right)=\frac{3}{4} \frac{9 \alpha C_{1} G^{2} M^{2}(2 G M-r)\left[\left(\frac{\alpha}{G M}\right)^{4} r^{2}+7\left(\frac{\alpha}{G M}\right)^{2} C_{1}^{2} r(r-2 G M)+4 C_{1}^{4}(2 G M-r)^{2}\right]}{\left(C_{1} \sqrt{1-\frac{2 G M}{r}}-\frac{\alpha}{G M}\right)^{6} \sqrt{1-\frac{2 G M}{r}} r^{12}} \\
& -\frac{3}{4} \frac{G^{3} M^{3}\left[2 C_{1}^{2}(2 G M-r)-\left(\frac{\alpha}{G M}\right)^{2} r\right]\left[\left(\frac{\alpha}{G M}\right)^{4} r^{2}-31\left(\frac{\alpha}{G M}\right)^{2} C_{1}^{2} r(2 G M-r)+4 C_{1}^{2}(2 G M-r)^{2}\right]}{\left(C_{1} \sqrt{1-\frac{2 G M}{r}}-\frac{\alpha}{G M}\right)^{6} r^{12}}, \\
& \operatorname{Im}\left(w_{2}\right)=0 .
\end{aligned}
$$

(c)

$$
\begin{aligned}
e^{\nu} & =A^{2} r^{2}, \quad e^{-\lambda}=\frac{1}{3}\left(1+\frac{B}{r^{2}}\right), \\
\text { RicciS } q & =\frac{2\left(2 B^{2}+r^{4}\right)}{3 r^{8}}, \quad \text { Riem } q=\frac{8\left(B^{2}+r^{4}\right)}{3 r^{8}}, \\
R & =0, \quad r_{1}=\frac{2 B^{2}+r^{4}}{6 r^{8}}, \quad r_{2}=\frac{4 B^{3}-3 B r^{4}-r^{6}}{36 r^{12}}, \\
\operatorname{Re}\left(w_{2}\right) & =\frac{1}{36 r^{6}}, \quad \operatorname{Im}\left(w_{2}\right)=0 .
\end{aligned}
$$

[1] L. Randall and R. Sundrum, Phys. Rev. Lett. 83, 3370 (1999); 83, 4690 (1999).

[2] P. Horava and E. Witten, Nucl. Phys. B460, 506 (1996).

[3] R. Maartens, gr-qc/0101059.

[4] T. Shiromizu, K. Maeda, and M. Sasaki, Phys. Rev. D 62, 024012 (2000).

[5] M. Sasaki, T. Shiromizu, and K. Maeda, Phys. Rev. D 62, 024008 (2000).

[6] K. Maeda and D. Wands, Phys. Rev. D 62, 124009 (2000); R. Maartens, ibid. 62, 084023 (2000); A. Campos and C.F. Sopuerta, ibid. 63, 104012 (2001); 64, 104011 (2001); C.-M. Chen, T. Harko, and M.K. Mak, ibid. 64, 044013 (2001); D. Langlois, Phys. Rev. Lett. 86, 2212 (2001); C.-M. Chen, T. Harko, and M.K. Mak, Phys. Rev. D 64, 124017 (2001); A. Coley, ibid. 66, 023512 (2002); Class. Quantum Grav. 19, L45 (2002); J.D. Barrow and R. Maartens, Phys. Lett. B 532, 153 (2002); H. Kudoh and T. Tanaka, Phys. Rev. D 65, 104034 (2002); H.A. Bridgman, K.A. Malik, and D. Wands, ibid. 65, 043502 (2002); C.-M. Chen, T. Harko, W.F. Kao, and M.K.
Mak, Nucl. Phys. B636, 159 (2002); M.K. Mak and T. Harko, Class. Quantum Grav. 20, 407 (2003).

[7] N. Dadhich and S.G. Ghosh, Phys. Lett. B 518, 1 (2001); M.G. Santos, F. Vernizzi, and P.G. Ferreira, Phys. Rev. D 64, 063506 (2001); M. Bruni, C. Germani, and R. Maartens, Phys. Rev. Lett. 87, 231302 (2001); H.-C. Kim, S.-H. Moon, and J.H. Yee, J. High Energy Phys. 02, 046 (2002); M. Govender and N. Dadhich, Phys. Lett. B 538, 233 (2002); T. Wiseman, Class. Quantum Grav. 19, 3083 (2002); R. Neves and C. Vaz, Phys. Rev. D 66, 124002 (2002); H. Kudoh, T. Tanaka, and T. Nakamura, ibid. 68, 024035 (2003).

[8] N. Dadhich, R. Maartens, P. Papadopoulos, and V. Rezania, Phys. Lett. B 487, 1 (2000).

[9] C. Germani and R. Maartens, Phys. Rev. D 64, 124010 (2001).

[10] R. Casadio, A. Fabbri, and L. Mazzacurati, Phys. Rev. D 65, 084040 (2002).

[11] S. Shankaranarayanan and N. Dadhich, gr-qc/0306111.

[12] M. Visser and D.L. Wiltshire, Phys. Rev. D 67, 104004 (2003).

[13] R. Casadio and L. Mazzacurati, Mod. Phys. Lett. A 18, 651 (2003). 
[14] S. Chandrasekhar, An Introduction to the Study of Stellar Structure (Dover Publications, New York, 1959).

[15] M.K. Mak, H.W. Chan, and T. Harko, Comput. Math. Appl. 41, 1395 (2001); M.K. Mak and T. Harko, ibid. 43, 91 (2002); T. Harko and M.K. Mak, ibid. 46, 849 (2003).

[16] L. Herrera and J. Ponce de Leon, J. Math. Phys. 26, 2303 (1985); 26, 2018 (1985); 26, 778 (1985); M.K. Mak and T. Harko, Int. J. Mod. Phys. D (to be published), gr-qc/0309069.

[17] P. T. Olver, Applications of Lie Groups to Differential Equations (Springer-Verlag, New York, 1993); B.J. Cantwell, Introduction to Symmetry Analysis (Cambridge University Press, Cambridge, 2002).
[18] C.B. Collins, J. Math. Phys. 18, 1374 (1977).

[19] K. Santusuosso, D. Pollney, N. Pelavas, P. Musgrave, and K. Lake, Comput. Phys. Commun. 115, 381 (1998).

[20] E. Zakhary and J. Carminati, J. Math. Phys. 42, 1474 (2001); J. Carminati, E. Zakhary, and R.G. McLenaghan, ibid. 43, 492 (2002).

[21] J. Garriga and T. Tanaka, Phys. Rev. Lett. 84, 2778 (2000); S. Nojiri and S.D. Odintsov, Phys. Lett. B 548, 215 (2002); M. Ito, ibid. 528, 269 (2002); 554, 180 (2003); D.K. Park, ibid. 562, 316 (2003); D.K. Park and S. Tamaryan, ibid. 554, 92 (2003). 